\title{
Enhanced URLLC-Enabled Edge Computing Framework for Device-Level Innovation in 6G
}

\author{
Syed Junaid Nawaz ${ }^{\ddagger}$, Shree Krishna Sharma ${ }^{\dagger}$, Mohmammad N. Patwary ${ }^{\S}$, and Md Asaduzzaman* \\ †Department of Electrical Engineering, COMSATS University Islamabad, Islamabad 45550, Pakistan. \\ ${ }^{\dagger}$ SnT - securityandtrust.lu, University of Luxembourg, Kirchberg, Luxembourg 1855, Luxembourg. \\ $\S$ Faculty of Science \& Engineering, University of Wolverhampton, Wolverhampton WV1 1LY, UK. \\ * School of Digital, Technologies and Arts, Staffordshire University, Stoke on Trent ST4 2DE, UK. \\ e-mail: junaidnawaz@ieee.org,shree.sharma@uni.lu,patwary@wlv.ac.uk, and asad@ieee.org
}

\begin{abstract}
The upcoming beyond 5G (B5G) wireless networks target various innovative technologies, services, and interfaces such as edge computing (EC), ultra-reliable and low-latency communication (URLLC), backscatter communications, and TeraHertz (THz) technology-enabled inter-chip communications, and high capacity links. Although there are ongoing advances in the system/network level, it is crucial to introduce innovations at the device-level to efficiently support these novel technologies by addressing various practical constraints such as power limitations, computational capacity, and storage capacity. Considering the contemporary latency requirements of future consumer electronics (CE) (e.g., entertainment, gaming, etc), to enhance the commercial potential of "edge processing as service", it is envisioned that URLLC will further evolve as enhanced-URLLC (e-URLLC). In this regard, this paper proposes a novel ECenabled e-URLLC framework for the next-generation CE devices, named EC for CE (ECCE), in order to enable the support of e-URLLC in the wireless $6 \mathrm{G}$ era. Starting with the discussion on recent trends and advances in $\mathrm{CE}$, the proposed framework and its importance in the $6 \mathrm{G}$ era are described. Subsequently, several potential enabling technologies and tools for the implementation of the proposed ECCE framework are identified along with a discussion on some interesting open research topics.
\end{abstract}

Keywords: 5G, 6G, edge computing, electronics, URLLC

\section{INTRODUCTION}

Towards enhancing the quality of human life experience, research in different areas is progressively contributing. Advancement in the areas of integrated circuits, information processing, and wireless communications towards boosting the number of transistors in the wafer area, speed of information processing, and rate of information exchange, respectively, is the prime catalyst delivering new types of services to the society. In the recent few years, the area of wireless communications has witnessed a remarkable advancement which has completely revolutionized the way we attend our daily activities. With the advent of $5^{\text {th }}$ Generation $(5 \mathrm{G})$ of wireless networks, a huge proliferation in the scale and types of network devices, products, services, applications, and businesses is anticipated. This tremendous expansion offered by $5 \mathrm{G}$ is envisioned to play a vital role in the advancement of society and the expansion of world economy. The revolutionary 5G technologies are predicted to contribute about $\$ 2.2$ trillion to the global economy in the next 14 years [1]. To bolster this economic revolution, a paradigm shift in the consumer elec- tronics $(\mathrm{CE})$ industry for beyond $5 \mathrm{G}(\mathrm{B} 5 \mathrm{G})$ and $6^{\text {th }}$ Generation (6G) wireless eras is imminent.

Rapid evolution and revolution in the CE industry have been witnessed in the recent years. However, the CE industry is currently in a rough transition period where effective design, installation, utilization, and predictive-maintenance of electronics devices at a massively large scale in the B5G wireless era is predicted to emerge as an immense challenge. The natural performance boundaries of the existing technologies compared to developing new diverse and contradicting performance and design requirements for the future CE plead the necessity of a huge leap forward in the CE framework. Such stringent requirements in next-generation CE include context-awareness, light-weighted devices, communications ability with lower overhead, intelligence provision, automation, security and privacy provisions, high energy-efficiency, low-cost, holographic, remote-interaction, haptic interaction, assurance of low human electromagnetic radiations exposure, multi-connectivity, ultra-high reliability, ultra-low latency, massive access, etc. Along with these rigorous requirements, ensuring barrier-free (universal) design of products, services, and environments is another prime challenge for the CE industry. Furthermore, the rapidly increasing demands of computational, storage, and communication abilities with the assurance of low-cost and high energy-efficiency of the devices is a challenging factor that needs a new framework to deliver the future quality-oflife-experience demands of the societies.

The potential of cloud services to support the growing demands of seamless computing and storage requirements are widely acknowledged. However, the high network latency in service delivery is its primary limitation. Now, with the advent of the ultra-reliable low-latency communications (URLLC) feature in $5 \mathrm{G}$, offering $10^{-9}$ packet-error-rate (PER) with $1 \mathrm{~ms}$ end-to-end (E2E) latency, this constraint is significantly resolved. Further evolution in URLLC towards enhancedURLLC (e-URLLC) is anticipated in B5G/6G (in a decade or so) to realize $0.01 \mathrm{~ms}$ E2E latency with $99.99999 \%$ reliability [2]. This inclusive succession holds the potential to revolutionize the existing wireless $\mathrm{CE}$ framework through the provision of e-URLLC connectivity of the devices to the edge computing (EC), edge storage, and other edge facilities. In this paper, the state-of-the-art of wireless CE is reviewed where 
future device types, device design requirements, performance requirements, related $5 \mathrm{G}$ technologies, associated socioeconomic aspects, and vision for next-generation $\mathrm{CE}$ in the $6 \mathrm{G}$ era are highlighted. A novel EC enabled e-URLLC framework for CE devices, named as EC for CE (ECCE), is proposed and its potential enabling techniques/tools are reviewed. The notion of "processing as a service" through e-URLLC is built. A low-cost, energy-efficient, and processor-less (minimal processor) device-level design is motivated. Open research topics and future recommendations for next-generation $\mathrm{CE}$ in the $6 \mathrm{G}$ era are discussed. The rest of this paper is organized as follows. Sec. II provides an outlook of need for devicelevel innovation in 6G. Sec. III presents the proposed ECCE framework. Sec. IV identifies the enabling techniques for the proposed framework and discusses the related open research topics. Finally, Sec V concludes the paper.

\section{Need for Device-Level Innovation in B5G/6G}

The standardization of $5 \mathrm{G}$ wireless networks commenced in 2019 with the Rel-15 of $3^{\text {rd }}$ generation partnership project (3GPP). 5G took a tremendous leap forward by offering various innovative technologies and services. The primary 5G services are enhanced mobile broadband (eMBB),URLLC, and massive machine-type communications (mMTC) [3]. 5G New Radio (NR) is still in its initial stage of development and it is expected to significantly evolve in the upcoming releases of 3GPP in B5G era. To fully consume the potential of anticipated B5G technologies and to meet the increasingly rigorous demands of future wireless networks along with the assurance of environmental protection, there is an immense need for device-level innovation in 6G.

In the 6G wireless era, by the year 2030, the global mobile and machine type subscribers are predicted to rise to about $17.1 \mathrm{bn}$ and 97bn [4], respectively. Various new types of devices are expected to emerge, e.g., wearable, body implants, textile embedded, human-machine interaction, smart cars, haptic sensors, smart home/office, network edge, consumer cognitive systems, and robots, etc. In a massively connected world of the future, a huge amount of data will be generated and communicated across device-, edge-, and network-levels of the communication skeleton. A 55\% annual increase in mobile data traffic is forecasted for the years from 2020 to 2030 [4]. This predicted voluminous amount of data generation can be effectively utilized for creating new services, improving the grade-of-service (GoS), and raising the value generation opportunities provided that a matching device-, edge-, and network-level innovative design in privileged. In the provision of "access to information at the fingertips", meeting the insatiable processing-power demands at the device-, edge, and network-sides requires a significant enhancement in the computing framework. With the anticipated massive increase in the number of devices, an increase in the chore of managing data across multiple heterogeneous devices (i.e., synchronization of contents) for the users will also arise. Moreover, the provision of high processing and storage capability for the devices with high energy efficiency and low cost is a prime

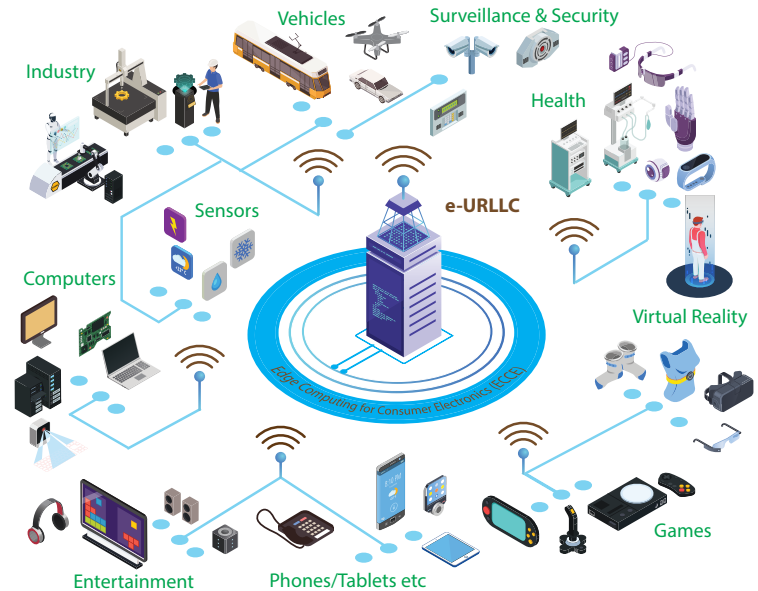

Fig. 1. Example CE device types and EC platform (i.e., ECCE).

research challenge for the $\mathrm{B} 5 \mathrm{G} / 6 \mathrm{G}$ wireless era. The advent of $5 \mathrm{G}$ technologies has extended new opportunities to rethink the existing computational and storage models as well as realize new business opportunities. The provisions of information exchange with ultra-low latency and ultra-high reliability (i.e., $10^{-9}$ PER with $1 \mathrm{~ms}$ E2E latency $[2,3]$ ) and the advent of the architectural concept of EC in 5G, besides supporting many dynamic applications, open new doors of opportunities for rethinking the device- and system-level architectures in nextgeneration CE.

Humans in the B5G digital society are expected to see enormous advancements in the types and quantity of network devices for augmenting the effective creation, processing, exchange, storage, and utilization of information. The use of smartphones, tablets, and computers may continue with some added features, while a boom in the device types for $\mathrm{B} 5 \mathrm{G} / 6 \mathrm{G}$ wireless networks is anticipated. The mMTC services of $5 \mathrm{G}$ are expected to revolutionize various areas of the future World through extending the provisions of sensing, communicating, and actuating in machine-type devices, e.g., the smart-industries, -cities, -homes/offices, -healthcare, agriculture, -mobility, etc. Furthermore, various context-aware and wearable devices for applications and services ranging from healthcare to seamless human-machine interaction are expected to emerge. For example, such devices may include bio-nano in-body devices, brain sensors, earpieces, textile embedded devices, surveillance and security devices, vehicular devices $(\geq 100 \mathrm{~km} / \mathrm{h})$, etc. The augmentation in machine learning (ML) and wireless communication methods combined with a plethora of such devices has the potential to signify intuitive and efficient interaction between humans and machines. Considering the nature, requirements, and constraints of such devices, it may not be attainable to furnish such devices with substantial battery and computing capabilities. In this context, offloading of the devices' computing tasks to the available computing resources at the network edge and/or network cloud through fast, reliable, and energy-efficient communication links is an attractive proposal to support the progression.

Today, CE products are more than just technology innovations and have become a vital influencing player in 

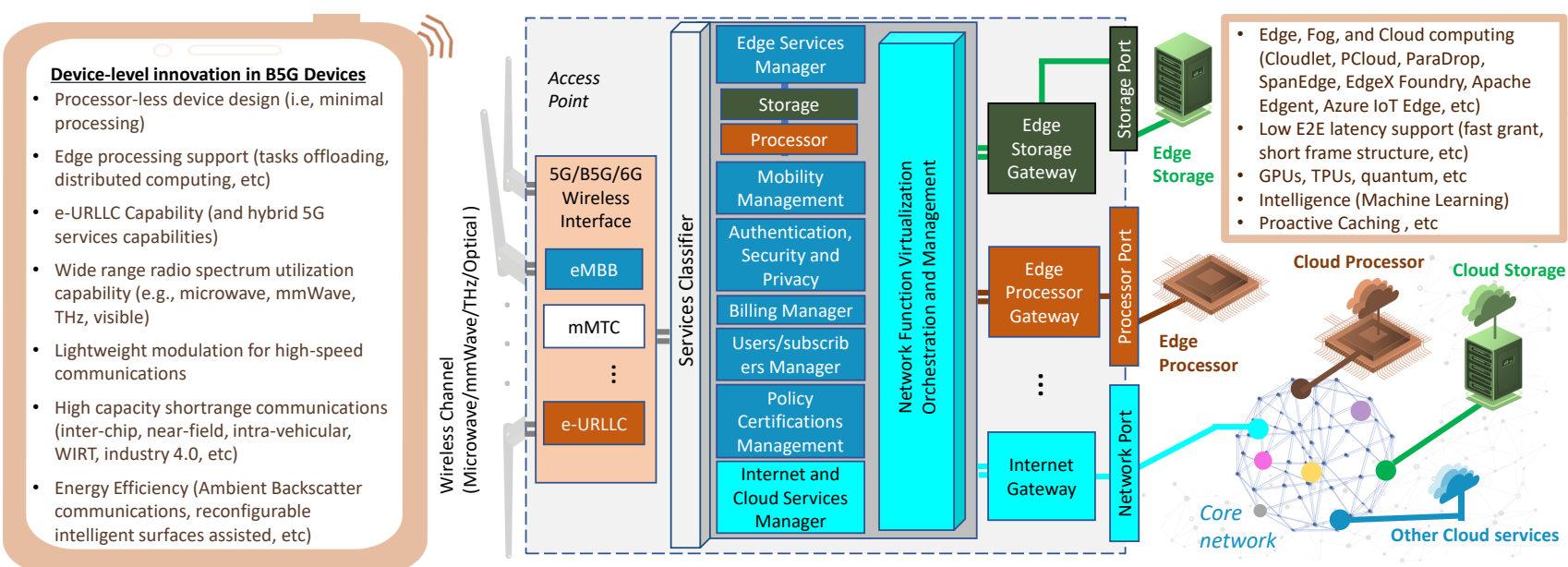

Fig. 2. Access point architecture for hosting the proposed ECCE platform with the indication of some key device- and system-level features.

the ecosystem of socioeconomics. This marks the electronic engineers as the architects of tomorrow with the responsibility to address various dynamic challenges even beyond the realm of science and engineering. The future wireless networks are expected to play a vital role in the socioeconomic ecosystem. Communication networks are becoming a central platform for daily life services delivery. Eventually, when all (most of) the daily life services will be delivered through the communication networks platform, the performance of wireless links will directly influence the overall grade of service delivery as well as socioeconomic aspects. Therefore, the quality-of-experience of the services by the consumers is required to be more comprehensively quantified with a holistic view of the impact and performance of each aspect.

\section{Proposed Framework for Device-Level INNOVATION IN B5G/6G}

The URLLC and EC features of $5 \mathrm{G}$ provide the industry and research communities with the privilege to rethink innovative use-cases for realizing their full potential. The future generation of wireless networks (i.e., B5G/6G) is expected to support significantly high-capacity and low-latency communications. To this end, it can be envisaged that future $\mathrm{CE}$ will be at the forefront of innovation to consume the offered wireless capabilities in the B5G/6G era. Considering the contemporary latency requirements of CE (e.g., entertainment, gaming, etc.), to enhance the commercial potential of "processing as a service", the research community intends to commit to the evolution of URLLC towards e-URLLC in the B5G/6G era. This succession is determined to achieve a reliability of $99.99999 \%$ with $0.01 \mathrm{~ms}$ E2E latency in a decade or so [2]. Such ultra-high reliability and ultra-low latency abilities can amply facilitate the device-design in terms of device-cost to support a wide range of $\mathrm{CE}$ devices. The processing capabilities available at the edge can be provisioned to the devices to support processor-less (or minimal-processor) device design. The basic concept of the proposed ECCE framework to deliver ondemand computing services to CE devices through e-URLLC services is illustrated in Fig. 1, where various device-type are also shown. The devices can rent the most expensive part "the computational processor" as an "edge processing service" on a "pay as you go" basis through an on-demand e-URLLC wireless connectivity. Appropriate modeling of such a demand and device-design is vital to serve a wide range of processing units and latency demands. The latency involved in offloading of computing tasks from the devices to an EC platform is expected to become a matching case to that of performing the tasks locally at the devices in the B5G/6G era.

The proposed ECCE platform can be hosted at the network access points (e.g., evolved node B (eNB), radio network controller (RNC), wireless fidelity (WiFi) routers, etc). The architecture of such access points and key device-level design features are shown in Fig. 2. The employed Services Classifier at the access points classifies and diverts the traffic/data received from the $5 \mathrm{G} / \mathrm{B} 5 \mathrm{G} / 6 \mathrm{G}$ radio interface to an appropriate forum, e.g., ECCE, cloud services, or internet platforms, without any loss in performance for network data users. For example, this can be achieved through bundling the traffic/data with the operation that is requested on that data (i.e., encapsulation of packets). Afterward, the respective service manager handles the subsequent operations; e.g., the Edge Services Manager (see Fig. 2) manages the edge services (computing, storage, etc) operations. A gateway provides access to each service type through the respective port/interface, e.g., Edge Storage Gateway, Edge processor Gateway, Internet Gateway, etc. Given the robust processing capability available at the network edge (e.g., at access points), along with enhancing the network experience for the mobile users it can also support the CE devices in their local computational tasks. The processing-time of the computational tasks of the $\mathrm{CE}$ devices can be reduced through their processing at a powerful processor interfaced at the edge, however, the latency of wireless links from the CE devices to the edge platform is critical in enhancing the overall performance tradeoff. Mobility management, authentication, security, privacy, policy certification, subscription, billing, operations can be looked after by the respective operational blocks, as indicated in Fig. 2.

The proposed ECCE concept facilitates improvements in energy efficiency (to enhance their battery life) demands 
of an exponentially growing number of wireless devices. This ultimately aids in building an environmentally friendly consumer device eco-system to help the climate. For the devices relieved from the burden of performing heavy computational tasks locally, the energy efficiency trade-off between the energy saved at the device-level in the account of reduced computational tasks and the energy consumed on communicating the tasks to the ECCE platform through eURLLC links is critical. Deploying multiple ECCE platforms and ensuring appropriate association of CE devices with the platforms can help in further enhancing the overall latency and energy efficiency. The window of opportunity provisioned by the proposed ECCE platform in terms of optimizing the overall energy consumption, device cost, and $\mathrm{CO} 2$ emission count trade-off is illustrated in Fig. 3. With the EC platform equipped with heavy processing capabilities, the device design can be provisioned with minimal processing capabilities resulting in significantly reduced device cost. For employing the proposed ECCE platform, the cost of the edge-side will increase, however, the cost per connected device (to ECCE) will reduce in the context of a massively connected network of the future (as the local processors in the devices usually stay in idle state). The device-level innovation required to exploit such a framework includes the provisions for hybrid $5 \mathrm{G}$ services, processor-less design (minimal processor), the ability to utilize a wide range of radio spectrum, etc. Rethinking of the access point design is required to add the support for connectivity for external processors through parallel ports/slots where processors of different capabilities can be plugged in. For such access-points/network-edge design, an appropriate external processor for a certain use-case can be installed, e.g., a heavy-duty processor for the laboratories to handle the tasks of all the connected wireless devices, etc. Furthermore, such an EC framework can also facilitate the CE devices to benefit from various emerging revolutionary processing and learning technologies, e.g., quantum computing, quantum ML, etc, through the integration of such facilities at the edge.

The proposed ECCE framework also opens new business opportunities for the network operators through the lending of on-demand processing services to the wireless connected $\mathrm{CE}$ devices. Such a service can be termed as "processing as a service" and it has immense potential in facilitating devicelevel innovation. The use-cases of this concept include: the access point in a cafe equipped with a heavy-duty processor for extending on-demand computing service to the user devices through e-URLLC services, etc. Such a service may be purchased by the users for a certain time duration or a certain amount of computational tasks as prepaid cards or codes from the cafe reception. Similarly, in research laboratories, libraries, hospitals, offices, homes, and public waiting areas, such ondemand services can be provided to the devices to offload their computational tasks. It can be, thus, envisaged that edgecomputing together with e-URLLC for CE devices, besides supporting mission-critical applications, can facilitate various new-types of services to the network subscribers, enterprise users, and allied industries in the B5G/6G era.

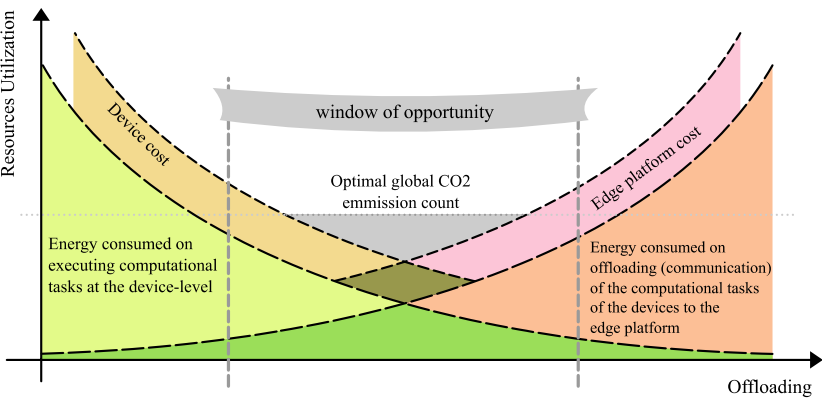

Fig. 3. ECCE supported energy and cost efficiency trade-off.

\section{Key EnABlers and Future ReCOMmEndations}

The key enabling technologies/tools to exploit the full potential of proposed ECCE framework are discussed in this section. In Fig. 4, a taxonomy of the key enablers and proposed innovations at the device- and system-levels is presented. The need of new business models for network edge operators to serve the notion of "processing as a service" is also indicated.

1) EC as a Service: The current trend in analyzing the massive amount of data from IoT devices is towards IoT-cloud convergence, in which a centralized cloud server processes the huge amount of IoT/sensor data and stores for future usage. Although, cloud computing is an excellent platform to handle and store the huge amount of data generated from the IoT systems, this is not suitable for applications demanding realtime operation and high Quality of Service (QoS) requirements [5]. To address this issue, EC/fog computing is considered an important enabler due to its offered benefits $[5,6]$, which include: (i) Proximity: Processing closer to the devices (at the eNBs or aggregation points), (ii) Lower Latency: considerable latency reduction is possible, (iii) Location awareness and real-time access to radio network and context information, (iv) high bandwidth availability at the edge and reduction of backhaul bandwidth. Various computing and storage nodes can be distributed across the network, with the IoT-devices/users on one side and cloud-center on the other side.

2) EC from Business Perspectives: The widely followed business model in the cloud-based IoT data analytics is a high fee pay-as-you-go business model. This model can not satisfy real-time requirements of latency-sensitive applications and also it creates data privacy concerns. This has led to the need of shifting data analytic functionalities to the edge of the network. Various sensors and machine-type devices such as industrial machines, smart home equipments, medical devices, smart meters, built-in sensors in mobile phones, planes, cars, and body area sensors produce a massive amount of data to be processed in the real-time. However, the existing cloud-centric architecture adopts distributed streaming architecture to collect and process the data at the cloud, and is not suitable to meet real-time constraints of latency-sensitive IoT applications. Also, the "pay-as-you-go" business model adopted by this architecture is not suitable for the end-users from the cost and ever-increasing data-volume perspective. Furthermore, the current cloud-centric architecture is not suitable to maintain the data privacy of local communities and people [7]. Moreover, 


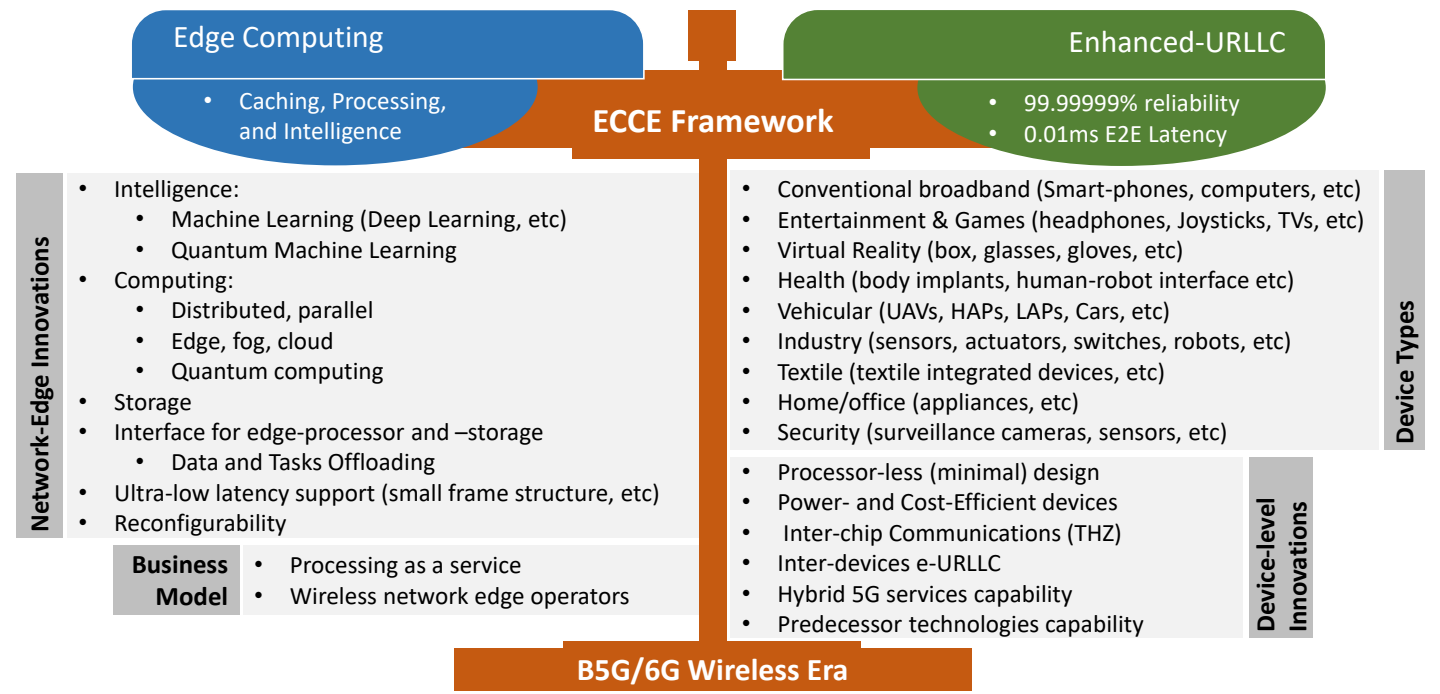

Fig. 4. The e-URLLC supported EC for wireless consumer electronics (ECCE) framework for device- and system-level innovation in 6G.

existing edge analytic techniques are not flexible to support the domain-specific processing of edge IoT applications.

3) Device-level Energy Efficiency: Minimizing energy consumption at the devices and edge-servers is crucial for enhancing the energy efficiency of $6 \mathrm{G}$ wireless networks. On one hand, due to several components involved in user devices such as operating systems, apps, and screen display consume a significant amount of the available energy budget at the device-side. On the other hand, resource-constrained nature of low-end sensors and IoT devices are not able to employ energy-hungry or computationally intensive algorithms at the device-side, leading to the need of energy saving mechanisms at the device-side. The energy consumed by a device per transmitted bit in 5G NR, in general, is minimized by enabling the higher data rates and lower latency as this facilitates to terminate the data sessions faster than in the LTE case. Nevertheless, due to several new features included in 5G NR such as shorter time slots, wider bandwidths and multiple scheduling events per time slot, the energy consumption at the device-level increases, and to address this, 3GPP Rel-15 specifications has proposed various energy saving mechanisms such as customized control channel monitoring, connectedmode Discontinuous Reception (cDRX) and inactive state. Furthermore, several additional features are being included for device energy savings in 3GPP Rel-16, e.g., dynamic cross-slot scheduling, dynamic SS adaptation, connected-mode wakeup signal, secondary cell dormancy, and device assistance for secondary cell release. By configuring the cDRX and Search Space (SS) parameters, network vendors can balance the tradeoff between device energy efficiency and system performance metrics such as latency and throughput [Ericsson].

Reflective technologies such as backscatter Communications (BsC) and Large Intelligent Surfaces (LIS) are the emerging paradigms to deliver the energy efficiency requirements of $6 \mathrm{G}$ wireless era. $\mathrm{BsC}$ has a huge potential to address the issues of limited battery life, cost of power and spectrum resources, and avoid the tedious task of recharging/replacing the batteries in battery-operated IoT devices, by enabling the deployment of battery-less passive devices in massive-IoT networks [8]. LIS can enable a programmable and controllable smart radio environment, which itself can be used as an added degree of freedom for optimizing the communication networks.

4) High Capacity Short Range Communications: Recent advances in cost-effective Complementary metal oxide semiconductor (CMOS) IC technologies and Terahertz (THz) waves $(300 \mathrm{GHz}-3 \mathrm{THz})$ have gained attention for a widerange of daily-life applications ranging from 3D imaging and electronic smelling to space communication networks. Towards enabling $\mathrm{THz}$ communications for $6 \mathrm{G}$ networks, research community is investigating various aspects of the required software and hardware modules including $\mathrm{THz}$ channel estimation, $\mathrm{THz}$ beam tracking, $\mathrm{THz}$ modulators, $\mathrm{THz}$ solid state superheterodyne receiver, and $\mathrm{THz}$ antennas, etc. $\mathrm{THz}$ communications can be broadly categorized into: (i) solid state $\mathrm{THz}$ wireless comm. system, which is based on frequency mixing mechanism, and (ii) direct modulation $\mathrm{THz}$ wireless systems, which is based on the modulation of the baseband signals directly to the RF. Despite promising benefits of $\mathrm{THz}$ communications, there are several challenges such as developing THz RF devices due to the required non-linear modeling and parameter extraction, supporting the required modulation depth and speed for the $\mathrm{THz}$ modulators, need of compact planar patch antennas, and low-complexity signal processing. The other prominent research directions include Industry 4.0 (no cabling requirements), $\mathrm{THz}$ communications for inter-chip communications [9], Near field communications and microbeamforming, and intra-vehicle/-car/-avionics communications (short-range wireless asynchronous real time (WIRT)) [10].

5) Tasks/Data Offloading and Distributed Computing: To provide seamless and low latency services to the consumer and enterprise end-users for a wide variety of devices, a suitable framework to handle an enormous amount of data and computational tasks will be required. In this regard, two promising emerging solutions are: (i) performing computations and data analytic operations in a distributed framework through edge servers within a certain geographic location, (ii) offloading 
the heavy computations and massive data to the edge or cloud servers. There exist several edge-computing deployment proposals in the literature which include: OCTEON Fusion Family deployed on a small-cell base station (BS), Nokia's software solution in BS, Cisco's IOx in service router, and Intel's Smart Cell Platform, etc [11]. A guaranteed low latency is obligatory in seamless offloading of the computational tasks. Many latency-sensitive real-time applications require a latency of $\leq 50 \mathrm{~ms}$ response time such as devices for visual guidance, online gaming, etc [11]. To this end, employing distributed EC servers can potentially provide an efficient solution. Although the distributed computing framework has been researched for over three decades, more dedicated efforts are still required to develop novel methods to support its EC applications.

6) Use-cases, Tools, and Models for EC: EC has promoted various interesting applications such as industrial automation, remote monitoring, autonomous vehicles, augmented reality (AR) and VR, Tele-medicine, connected homes and offices, smart agriculture, predictive maintenance, video monitoring, blockchain, and smart cities. The notable use-cases of EC include: IoT data/tasks offloading (sensors-to-edge, edge-tocloud, etc), decentralized network orchestration (autonomic functioning of edge nodes in case of backhaul link failure, edge caching, etc), formation of localized networks (localized services, location-aware resource allocation, etc.), and factory and enterprise communication provisions (industrial and enterprise IoT, URLLC, security, etc). Based on the innovation in architecture, programming tools and applications, there are many EC systems and tools proposed so far, viz: Cloudlet, CloudPath, PCloud, ParaDrop, SpanEdge, Cloud-Sea Computing Systems, Cachier and Precog, FocusStack, AirBox, Firework and open source projects: CORD (Central Office Re-architected as a Datacenter), Akraino Edge Stack, EdgeX Foundry, Apache Edgent, Azure IoT Edge [12]. Depending on the type of target devices, edge nodes, computational tools, each of these EC tools/systems vary in design and features. Alongside the discussed developments in variety of developed systems and tools, the idea of EC is also evolving for decentralization of the cloud resources towards the enhancement of EC capabilities. Various simulation and emulation tools are available to realize and deploy the concept of edge/fog computing including FogNetSim++, iFogSim, FogTorchII, EdgeCloudSim, IoTSim, EmuFog, Fogbed, NS2/NS-3, Graphics Processing Unit (GPU), High Performance Computing (HPC), and Software Defined Radio (SDR). In order to connect edge-devices and process data at the edge, various IoT platforms including Arkessa, ThingSquare and ThingWorx exist. Similarly, various lightweight messaging protocols such as constrained application protocol (CoAP) and messaging queue telemetry transport (MQTT) are designed to effectively collect and process the data from IoT devices [7].

7) Quantum Computing and Communications: The quantum mechanics concepts are envisioned to revolutionize various sectors of daily life including computing and communications [3]. The CE devices built from elementary particles are subject to exercise the principles of quantum mechanics enduring a strong association with the postulates of Euclidean geometry. The fundamental concepts of quantum superposition, quantum entanglement, or the no-cloning theorem offer an immense amount of parallelism in the computing concepts, which has also received the endorsement through recently demonstrated results. Quantum computing systems can effectively generate counter-intuitive statistical data patterns that are beyond the reach of classical computing systems [3]. As recognition of these potentials, new disciplines like "Quantum Computer Science" and "Quantum Information Science" have also originated. In the exploitation of these physical quantum mechanics phenomena, a revolution in communications and computing sectors is envisioned to be around the corner [3].

\section{Conclusions}

Starting with the review of the state-of-the-art of $\mathrm{CE}$, an EC-enabled e-URLLC framework to support device-level innovation in the $6 \mathrm{G}$ era has been proposed. Notable enablers for the proposed framework have been identified and reviewed. Built on the anticipated evolution in 5G technologies, a notion of "computing as a service" has been manifested. A low-cost, energy-efficient, and processor-less (minimal) device-design has been motivated. Open research topics and future recommendations for the next-generation $\mathrm{CE}$ in the $\mathrm{B} 5 \mathrm{G} / 6 \mathrm{G}$ wireless era have been discussed. Various useful recommendations to drive future research work on the next-generation $\mathrm{CE}$ have been suggested. Furthermore, the value creation opportunities for network edge operators through renting of the EC facilities on "pay as you go" basis have also been discussed.

\section{REFERENCES}

[1] GSMA, "The mobile economy," Report, 2019.

[2] International Network Generations Roadmap (INGR), “Testbed,” IEEE Future Networks: Enabling 5G and Beyond, Report, 2019.

[3] S. J. Nawaz, S. K. Sharma, S. Wyne, M. N. Patwary, and M. Asaduzzaman, "Quantum machine learning for $6 \mathrm{G}$ communication networks: State-of-the-art and vision for the future," IEEE Access, vol. 7, pp. 46317-46350, 2019.

[4] I. T. U. (ITU), "IMT traffic estimates for the years 2020 to 2030," Report ITU-R M. 2370-0, ITU-R Radiocommunication Sector of ITU, 2015.

[5] S. K. Sharma and X. Wang, "Live data analytics with collaborative edge and cloud processing in wireless IoT networks," IEEE Access, vol. 5, pp. 4621-4635, 2017.

[6] A. Alnoman, S. K. Sharma, W. Ejaz, and A. Anpalagan, "Emerging edge computing technologies for distributed IoT systems," IEEE Network, vol. 33, no. 6, pp. 140-147, 2019.

[7] X. Xu, S. Huang, L. Feagan, Y. Chen, Y. Qiu, and Y. Wang, "EAaaS: Edge analytics as a service," in proc. of IEEE Int. Conf. Web Services, 2017, pp. 349-356.

[8] S. J. Nawaz, S. K. Sharma, B. Mansoor, M. N. Patwary, and N. M. Khan, "Non-coherent and backscatter communications: Enabling ultra-massive connectivity in the era beyond 5G," arXiv:2005.10937, 2020.

[9] Q. J. Gu, "THz interconnect: The last centimeter communication," IEEE Communications Magazine, vol. 53, no. 4, pp. 206-215, 2015.

[10] R. Adeogun, G. Berardinelli, P. E. Mogensen, I. Rodriguez, and M. Razzaghpour, "Towards $6 \mathrm{G}$ in-X subnetworks with sub-millisecond communication cycles and extreme reliability," IEEE Access, vol. 8, pp. $110172-110188,2020$.

[11] B. Varghese, N. Wang, S. Barbhuiya, P. Kilpatrick, and D. S. Nikolopoulos, "Challenges and opportunities in edge computing," in proc. of IEEE Int. Conf. Smart Cloud, 2016, pp. 20-26.

[12] F. Liu, G. Tang, Y. Li, Z. Cai, X. Zhang, and T. Zhou, "A survey on edge computing systems and tools," Proc. of the IEEE, vol. 107, no. 8, pp. 1537-1562, 2019. 